www. revistadyo.com

\title{
Estructurando el diseño de envases y embalajes para mejorar la sostenibilidad. Evidencias empíricas en el sector de menaje
}

\author{
Jesús García-Arca , A. Trinidad González-Portela Garrido, J. Carlos Prado-Prado e \\ Iria González-Romero
}

https://doi.org/10.37610/dyo.v0i73.593
Recibido: 10 de Noviembre de 2020

Aceptado: 07 de Enero de 2021

\section{Resumen}

En mercados cada vez más globales, volátiles y competitivos las empresas tienen que promover activamente acciones y políticas que mejoren la eficiencia y la sostenibilidad de sus cadenas de suministro. En este contexto, el adecuado diseño de envases y embalajes constituye un factor clave para lograr este objetivo. A finales del siglo XX empieza a conceptualizarse el enfoque "Packaging Logistics" que persigue la integración eficiente y sostenible de los sistemas de envase, embalaje, producto y cadena de suministro para lograr ventajas competitivas; dicho marco se amplía posteriormente para promover la mejora del comportamiento sostenible de empresas y cadenas de suministro con el enfoque "Sustainable Packaging Logistics" (SPL). En este contexto, en este artículo se busca validar conceptual y empíricamente hasta qué punto la aplicación de un modelo estructurado que despliegue el enfoque SPL puede contribuir de forma activa a un mejor comportamiento sostenible en la cadena de suministro. Para ello, se justifica y propone un modelo de despliegue que después se analiza en una muestra de 66 fabricantes del sector de menaje en España. Los resultados alcanzados validan mayoritariamente los aspectos del modelo propuesto

\section{Palabras clave}

Envase; Embalaje; Logística; Cadena de Suministro; Eficiencia; Sostenibilidad

\section{El diseño del envase y el embalaje en un contexto sostenible}

En mercados cada vez más turbulentos y volátiles, donde ya no se compite entre empresas sino entre cadenas de suministro, las organizaciones deberían diseñar e implantar iniciativas que incrementen su competitividad (Christopher, 2005). Así, las empresas se ven en la necesidad de emprender acciones que persigan el máximo rendimiento en las actividades realizadas a lo largo de toda su cadena de suministro (producción, transporte, manipulación, almacenamiento...), eliminando "despilfarros" o actividades que no añadan valor (enfoques "Kaizen" o "Lean Manufacturing") pero, también, desarrollando innovaciones en procesos, productos y servicios.

\footnotetext{
\esús García-Arca * jgarca@uvigo.es

iD ORCID: 0000-0002-3369-450X A. Trinidad González-Portela Garrido * tgonzalez-portela@uvigo.es

iD ORCID: 0000-0001-5329-0329 J. Carlos Prado-Prado * jcprado@uvigo.es

iD ORCID: 0000-0003-2189-2100 Iria González-Romero * iria.gonzalez.romero@uvigo.es

iD ORCID: 0000-0002-0225-363X

* Grupo de Ingeniería de Organización (GIO) Escuela de Ingeniería Industrial. Campus Lagoas-Marcosende. Universidad de Vigo, 36310, Vigo (España) .
}

Simultáneamente, diferentes "partes interesadas" (o "stakeholders") están mostrando un interés creciente por el desarrollo de la sostenibilidad (económica, medioambiental y social) aplicada a las cadenas de suministro, en línea con el fomento de actividades que fomenten la "Economía Circular". Si bien un número creciente de empresas están apostando por el desarrollo de cadenas de suministro sostenibles, todavía son mayoría las que se limitan a cumplir con sus compromisos legales a nivel individual, pero pasando de puntillas por su responsabilidad "extendida" aguas arriba y abajo en su cadena. No obstante, diferentes fuentes señalan el interés de desarrollar cadenas de suministro sostenibles, dado que permite ahorrar recursos, reducir residuos y niveles de contaminación y, simultáneamente, proporcionar ventajas competitivas (Seuring y Müller, 2008).

En este contexto, el diseño de envases y embalajes constituye uno de los elementos clave que pueden contribuir activamente a la mejora de la eficiencia y sostenibilidad de las cadenas de suministro (entre otros autores, Klevas, 2005; Hellström y Saghir, 2007; Garcia-Arca y Prado-Prado, 2008; Azzi et al., 2012; García-Arca et al., 2014, Hellström y Olsson, 2017; Palsson, 2018). Un adecuado diseño de los envases y embalajes puede ayudar a eliminar "despilfarros", tales como, el desaprovechamiento del espacio en almacenes, transportes y puntos de venta, la dificultad de manipulación de los productos, el exceso de materiales empleado, los rechazos y la pérdida de productividad en los procesos productivos de envasado, o las roturas/deterioros en los productos. Más aún, los propios envases y embalajes son 
fuente de innovaciones en productos, procesos y materiales, lo que también refuerza su aportación estratégica.

Ahora bien, este proceso de diseño del envase y el embalaje demanda una visión holística que permita la búsqueda e implantación de alternativas eficientes y sostenibles. Empresas internacionalmente conocidas como Ikea, Walmart, Proctel \& Gamble, Amazon o Inditex han hecho de sus envases y embalajes una piedra angular de sus estrategias comerciales y logísticas. Esta afirmación es válida no sólo para empresas que operan en el sector de consumo, sino también para aquellas que operan en el mercado industrial. La correcta integración del diseño del envase, el embalaje, el producto y la cadena de suministro en un marco general de sostenibilidad, es el fin último del enfoque "Packaging Logistics" (Saghir, 2002) y más recientemente del enfoque "Sustainable Packaging Logistics (SPL)" (García-Arca et al., 2014).

En el contexto, anterior el objetivo principal de este artículo es desarrollar y validar conceptual y empíricamente hasta qué punto la aplicación de un modelo estructurado que despliegue el enfoque SPL puede contribuir de forma activa a un mejor comportamiento sostenible en el conjunto de la cadena de suministro.

Con objeto de cumplir este objetivo, este artículo se estructura en cinco apartados. Así, tras este breve apartado introductorio, se realiza una síntesis de los principales aspectos a tener en consideración en el enfoque SPL, como parte intrínseca del modelo conceptual que se propone, basado en la revisión bibliográfica y en la reflexión $\mathrm{y}$ experiencia de los autores en proyectos aplicados en este campo. Posteriormente, se exponen los objetivos y la metodología con la que se analizará, de forma empírica, el modelo propuesto en una muestra de 66 empresas fabricantes del sector de menaje en España. A continuación, se dedica un apartado a la presentación de los resultados del estudio realizado. El artículo termina con un apartado de conclusiones.

La principal motivación de los autores para la realización del artículo está asociada con el hecho de que, aunque en la literatura reciente existe un amplio consenso conceptual en cuanto a la importancia del envase y embalaje como medio para mejorar el comportamiento sostenible de las cadenas de suministro, sin embargo, este tratamiento conceptual ha tenido un escaso análisis empírico (Azzi et al., 2012; Pålsson y Hellström, 2016; Wikström et al., 2018; Palsson y Sandberg, 2020; Freichel et al., 2020).

En este ámbito empírico, la literatura reciente se centra principalmente en el estudio de casos (Hellström y Nilsson, 2011; Kye et al., 2013; Accorsi et al., 2014; García-Arca et al., 2014; Pålsson y Hellström, 2016; Rundh, 2016; Sohrabpour et al., 2016; García-Arca et al., 2020) o en la comparación de envases y embalajes de diferentes productos (Svanes et al., 2010; Vernnuccio et al. 2010; Albretch et al.,
2013; Gelici-Zeco et al., 2012; Wever y Vogtländer, 2013; Bertoluci et al., 2014; Hellström y Olsson, 2017), más que en un análisis organizativo del despliegue del SPL con una muestra más amplia de empresas como la que se plantea en este artículo.

No obstante, existen en la literatura algunos estudios que plantean algunos de los aspectos del modelo propuesto para el despliegue del enfoque SPL; en este último ámbito, destacan los estudios en el sector alimentario (García-Arca et al., 2016), en el sector de juguetes (García-Arca et al., 2017) y en el sector de perfumería y productos de limpieza (García-Arca et al., 2019). Este artículo complementa los estudios anteriores dotándolos de un enfoque más completo e integrado en la interpretación aplicada del desarrollo del enfoque SPL.

\section{Estructurando el proceso de diseño de envases y embalajes}

Como paso previo a proponer y justificar el modelo de despliegue del enfoque "Sustainable Packaging Logistics" es necesario, no sólo conocer y comprender los diferentes requisitos de diseño que debe satisfacer el sistema de envase y embalaje, sino también sus implicaciones organizativas, así como la forma de comparar de forma objetiva las diferentes alternativas de diseño. A continuación, se desarrollan cada uno de estos aspectos.

\subsection{Requisitos de diseño del envase y el embalaje}

Más allá de la tradicional e imprescindible protección del producto, el envase y el embalaje deberían diseñarse, no sólo para proporcionar capacidad de diferenciación comercial, sino también para proporcionar eficiencia productiva/logística $y$, todo ello, en un contexto de desarrollo de la sostenibilidad en sus tres dimensiones (económica, medioambiental y social).

Así, la dimensión económica de la sostenibilidad podría analizarse desde dos perspectivas diferentes y complementarias: la de ingresos y la de costes. Por un lado, un adecuado diseño del envase y el embalaje puede constituir ese "vendedor silencioso" que resalte determinadas características (tangibles e intangibles) de los productos que incrementen su capacidad de diferenciación y que sorprendan positivamente al mercado; todo ello, contribuiría a mejorar las ventas. En este campo, los envases y embalajes juegan un rol activo en la innovación de nuevos productos, lo que todavía refuerza más este potencial comercial (Verghese y Lewis, 2007; Olander-Roese y Nilsson, 2009; Vernuccio et al., 2010; Hellström y Nilsson, 2011; Azzi, et al., 2012; Lindh et al., 2016).

Por otro lado, unos adecuados envases y embalajes pueden facilitar la reducción de los costes globales en la cadena de 
suministro (particularmente, los productivos y logísticos), aspecto crítico, a la hora de conceptualizar y comprender el impacto de ciertas decisiones de diseño. Autores como Hellström y Saghir (2007), García-Arca y Prado-Prado (2008), Svanes et al. (2010), Sohrabpour et al. (2012), Azzi et al. (2012), Kye et al. (2013) o Sohrabpour et al. (2016) plantean como requisitos de diseño en los envases y embalajes y para la reducción de los costes el que éstos faciliten una serie de tareas y procesos de la logística directa (desde los aprovisionamientos hasta el consumidor).

Cambiando el foco del análisis hacia la segunda de las dimensiones de la sostenibilidad (la medioambiental), el diseño de los envases y embalajes también afecta a un mayor o menor nivel de consumo de recursos. En este consumo, se incluyen los propios materiales de envases y embalajes, pero también otros recursos empleados a lo largo de la cadena de suministro como el combustible, necesario para el transporte de mercancías. Asimismo, el diseño de envases y embalaje también afecta a una mayor o menor generación de residuos y contaminación. Lógicamente, tampoco es menor el impacto medioambiental de los productos estropeados o deteriorados, motivados por un inadecuado diseño de envase y embalaje (Hellström y Saghir, 2007; Sohrapour et al., 2016; García-Arca et al., 2017).

La comprensión y sensibilización por este impacto medioambiental, particularmente a nivel de residuos $\mathrm{y}$ contaminación, ha supuesto el desarrollo de legislaciones específicas, entre las que destaca la Directiva Europea 94/62 (y su actualización 2004/12//EC), que han supuesto, en la práctica, la promoción del punto verde y la adopción de mecanismos específicos de gestión de envases y residuos de envases. Estas legislaciones, acordes con la creciente demanda de la sociedad en el campo medioambiental y con los objetivos de desarrollo sostenible marcados por las Naciones Unidas en 2030 (https://www.un.org/ sustainabledevelopment/es/objetivos-de-desarrollo-

sostenible) y con la promoción de la economía circular, fijan como prioridad conceptual la prevención en la generación de residuos de envases y embalajes (Svanes et al., 2010; Azzi et al., 2012; Albrecht et al., 2013; Grönman et al., 2013; Molina-Besch y Pålsson, 2014; de Koiejer et al., 2017, 1 y 2). Cuando este objetivo prioritario de prevención no sea factible, en segundo lugar, se persigue el aprovechamiento del residuo, empezando por la propia reutilización del envase y/o embalaje (Grönman et al, 2013). Continuando por orden de importancia, cuando la reutilización del residuo no sea posible, se promoverá el reciclado y la valorización de los residuos (incineración con generación de energía eléctrica).

Finalmente, el diseño de los envases y embalajes también tiene impacto en la dimensión social de la sostenibilidad. Entre otros aspectos asociados a esta dimensión de la sostenibilidad cabría citar: el facilitar las labores de reciclaje, el suministro de información honesta, comprensible y veraz, el garantizar la seguridad en el consumo del producto y la adaptación del uso, ergonomía y dosificaciones de producto a las necesidades de los diferentes clientes (por ejemplo, en personas de la tercera edad o con discapacidad) (Williams et al., 2008; Vernuccio et al., 2010; Azzi et al., 2012; GarcíaArca et al., 2017; Hellström y Olsson, 2017).

En base a todo lo expuesto anteriormente y en relación con la sostenibilidad se puede comprender el impacto y el carácter poliédrico de los requisitos de diseño que deberían satisfacer los envases y embalajes, los cuales se ubican en planos y necesidades muy diferentes. Estos diferentes requisitos de diseño se resumen en la tabla 1 que adapta la síntesis realizada por García-Arca et al. (2019) a partir de las aportaciones de autores como Azzi et al. (2012), Lindh et al. (2016), Rundh (2016) y Garcia-Arca et al. (2017).
Tabla 1 Requisitos de diseño de envases y embalajes.

\begin{tabular}{|c|c|}
\hline Requisitos diseño & Función principal \\
\hline Protectores & $\begin{array}{l}\text { Asegurar la adecuada protección del producto a lo largo de la cadena de suministro, } \\
\text { disminuyendo deterioros y roturas }\end{array}$ \\
\hline Logísticos & $\begin{array}{l}\text { Facilitar las tareas de manipulación, almacenaje y transporte a lo largo de la cadena de suministro } \\
\text { (logística directa e inversa; logística interna y externa) }\end{array}$ \\
\hline Compras & Garantizar y simplificar el proceso de compra y aprovisionamiento de los propios envases y embalajes \\
\hline Ergonómicos & $\begin{array}{l}\text { Facilitar la ergonomía en la manipulación del producto a lo largo de la cadena de } \\
\text { suministro, incluyendo los puntos de venta y el consumidor }\end{array}$ \\
\hline Legales & $\begin{array}{l}\text { Facilitar el cumplimiento de las normas y leyes asociadas al envase y embalaje (por ejemplo, } \\
\text { en productos químicos, farmacéuticos, alimentarios,...) }\end{array}$ \\
\hline Comunicación & $\begin{array}{l}\text { Proporcionar información útil y fiable a los diferentes usuarios de los envases y embalajes a lo largo } \\
\text { de la cadena de suministro, incluyendo puntos de venta y consumidores }\end{array}$ \\
\hline
\end{tabular}


No obstante, el conocimiento y comprensión de los múltiples y variados requisitos de diseño a los que están sometidos los envases y embalajes, siendo muy importantes, no es suficiente, dado que también es necesario conocer la propia estructura del sistema de envase y embalaje, típicamente estructurado en tres niveles (primario, secundario y terciario).

El envase primario (“primary packaging”) es el primer protector del producto y, típicamente, está en contacto con él; también se conoce con el nombre de "inner container" (contenedor interno), "first container" (primer contenedor) o "consumer package" (envase del consumidor). Este nivel, a su vez, puede contar simultáneamente con varios formatos, materiales y/o capas (por ejemplo, un bote en un estuche de cartón).

Por otro lado, el envase secundario está diseñado para agrupar varios envases primarios, del mismo o de diferente producto, otorgándole protección y facilidad de manipulación para su distribución comercial y venta. Habitualmente se desecha cuando se utiliza el producto o cuando se almacena en el hogar. Normalmente, este nivel se conoce con el nombre genérico de embalaje, "outer packaging" (envase externo) o "retail packaging" (envase de distribución). Este nivel también puede contar con varios formatos, materiales y/o capas (por ejemplo, una bandeja de cartón y un film plástico para agrupar un pack de botellas de agua o de briks de leche).

Finalmente, el último nivel del envase y embalaje es el envase terciario, el cual se asocia, típicamente, con varios envases secundarios (del mismo o diferente producto) que se agrupan, por ejemplo, en un palé o "roll container" para facilitar las tareas de manipulación, almacenaje y transporte. Este nivel de envase terciario también se conoce como unidad de carga ("unit load") o envase de transporte ("transport packaging").

El hecho de que tanto los envases secundarios como los envases terciarios puedan incluir uno o varios productos diferentes, está asociado a la necesidad de adecuarse a las necesidades de los pedidos de una empresa, tienda y/o cliente final (por ejemplo, un pedido de comercio electrónico).

Lógicamente, dado que estas agrupaciones multiproducto suelen romper con la configuración inicial fijada por la empresa envasadora, su materialización requiere actividades de manipulación adicionales que condicionan la eficiencia y sostenibilidad de la cadena de suministro en su conjunto. Asimismo, variables como el tamaño del producto, su fragilidad, el número de unidades que se comercializan y la frecuencia de los envíos también condicionan el diseño.

La interrelación entre los diferentes niveles del sistema de envase y embalaje (primario, secundario, terciario) debería ser coherente con los diferentes requisitos de diseño a los que están sometidos. Así, dado que existe una dependencia considerable entre los tres niveles para asegurar que se cumplen los requisitos de diseño, la adecuación de cada nivel no debería ser valorada de forma aislada sino, también, de forma conjunta e integrada con el resto de niveles. Además, esta visión integrada debería estar relacionada con las propias características y requisitos de diseño del producto y de su cadena de suministro asociada.

\subsection{Consideraciones organizativas en el diseño de los envases y embalajes}

Una vez descritos los requisitos de diseño sería necesario estructurar el propio proceso de diseño, esto es, profundizar en sus consideraciones organizativas. Lógicamente, la variedad de requisitos exige cierto nivel de coordinación interna y externa en el proceso de diseño. Asimismo, la importancia que se otorga a cada uno de los requisitos de diseño depende de qué área, departamento o empresa de la cadena de suministro lo valore. Además, estos requisitos de diseño no se reparten por igual en los diferentes niveles del sistema de envase y embalaje, pero tampoco lo hacen a nivel de cada etapa, proceso y tarea dentro de la cadena.

Por otro lado, las decisiones finales que afectan a los diferentes niveles del sistema de envase y embalaje (primario, secundario y terciario) también podrían estar relacionadas con la parte de la cadena de suministro que pueda ejercer una mayor influencia o liderazgo. Por ejemplo, si las empresas distribuidoras "dominan" a las empresas envasadoras (lo que es habitual, por ejemplo, en el sector de consumo), las decisiones finales relacionadas con los envases y embalajes podrían estar supeditadas a lo que el distribuidor considere prioritario. Lógicamente, este planteamiento no tiene por qué conducir necesariamente a la mejor solución de envase y embalaje desde una perspectiva global de cadena.

Por tanto, la estructura organizativa adoptada debería encargarse de tomar decisiones de diseño en los envases y embalajes de forma coordinada y consensuada entre las diferentes partes afectadas, esto es, diferentes áreas o departamentos dentro de cada empresa y diferentes empresas dentro de cadena de suministro (fabricantes de envases y embalajes, envasadoras, distribuidores, operadores logísticos, otros proveedores...). Asimismo, deseablemente, esta estructura organizativa también debería coordinarse e integrarse con los responsables de tomar decisiones en el diseño del producto y de la cadena de suministro.

Existirían, conceptualmente, diferentes planteamientos organizativos para afrontar de forma global la estructuración del proceso de diseño (Olander-Roese y Nilsson, 2009). Dentro de estas alternativas el más eficiente sería un enfoque "dinámico", donde el diseño del envase y embalaje lidera y promueve el diseño del propio producto (y de su cadena de suministro asociada). Cuando se aplica este enfoque 
"dinámico" se dispone, no sólo de un mayor número de alternativas eficientes y sostenibles, sino también un criterio ágil y coordinado para su selección, llegando a convertirse en el verdadero motor del cambio y de la mejora continua de los procesos desarrollados a lo largo de cadena de suministro. Este es el planteamiento propuesto en el enfoque "Sustainable Packaging Logistics" que se desarrolla posteriormente. En este contexto, a nivel de sistema de envase y embalaje, las decisiones de diseño que debería tomar esta estructura organizativa se centrarían en cinco aspectos complementarios y fuertemente interconectados (García-Arca et al., 2019):

- La selección de materiales a emplear, incluyendo tipología y calidades.

- La selección de las tecnologías relacionadas con los procesos donde intervienen los envases y embalajes. Entre estas tecnologías se encontrarían, por ejemplo, las adoptadas en los procesos de envasado y embalado o en los de manipulación, almacenaje y transporte incluyendo, en su caso, la selección de diferentes niveles de automatización. Asimismo, se encontrarían las tecnologías de identificación (como el RFID o los códigos de barras).

- El diseño estético del envase y del embalaje (forma, colores, textos, símbolos, imágenes...).

- $\quad$ El detalle de la estructura de niveles y agrupaciones del sistema de envase y embalaje (primario, secundario y terciario).

- La selección de las dimensiones empleadas en los envases y embalajes.

\subsection{Sistemas de valoración de alternativas de diseño en el envase y el embalaje}

En la literatura académica y empresarial se hace hincapié en la falta de métricas específicas para medir y comparar de forma global la eficiencia y sostenibilidad de las diferentes alternativas de diseño de envases y embalajes. La disponibilidad, sin embargo, de un adecuado sistema de valoración de estas alternativas constituye uno de los elementos críticos para determinar el diseño más adecuado. Así, parecería razonable catalogar el "mejor" envase y embalaje como aquel que, simultáneamente, combinase el diseño más atractivo y útil para el consumidor (final o industrial), con el mínimo coste global y generando el mínimo impacto en el medio ambiente (incluyendo la reducción de las pérdidas y deterioros de productos).

Ahora bien, tal como se ha comentado anteriormente, el envase y el embalaje está sometido a una diversidad de requisitos de diseño. Lógicamente, también pueden existir métricas diferentes asociadas al despliegue de cada uno de estos requisitos de diseño. Así, un adecuado diseño de envase y embalaje puede resaltar determinadas características de los productos, para incrementar su capacidad de diferenciación $\mathrm{y}$, por tanto, las ventas. En cualquier caso, las ventas constituyen en sí mismas un indicador "a posteriori", dado que miden esta idoneidad una vez implantados los nuevos formatos, por lo que no parece la forma más razonable para aproximarse al problema de partida (como medir los pros y contras de cada alternativa de diseño). Una forma de medir este impacto antes del lanzamiento de los nuevos formatos está asociada a técnicas como las pruebas de productos, la visibilidad o la estimulación en "laboratorio". Asimismo, se puede complementar con técnicas de comparativas de envases y embalajes de competidores ("Benchmarking"), aunque generalmente estas técnicas suelen dar prioridad a los aspectos comerciales a nivel del envase primario (GeliciZeco et al., 2012; Magnusson et al., 2012).

Por otro lado, un adecuado diseño de envases y embalajes puede facilitar la reducción de los costes globales en la cadena de suministro. Muchas empresas encuentran en este campo de la reducción de costes incentivos suficientes para promover cambios en sus envases y embalajes. Entre estos costes se podrían citar: los de compras y aprovisionamientos (reduciendo costes y garantizando suministros); los procesos de manipulación, almacenaje y transporte a lo largo de toda la cadena de suministro desde los proveedores y envasadores, hasta los distribuidores y puntos de venta (típicamente, asociados al concepto de costes logísticos, tanto internos en cada empresa, como externos en el resto de su cadena de suministro); los procesos productivos, típicamente asociados a las actividades de envasado y embalado (por ejemplo, facilitando las tareas de planificación y producción, simplificando los tiempos de preparación o reduciendo el índice de rechazos) (Morabito et al., 2000; Wever, 2011; Kye et al., 2013; García-Arca et al., 2014; Mejía-Argueta et al., 2015; Sohrabpour et al., 2016).

Lamentablemente, la relación de estos costes con las decisiones de diseño de envases y embalajes presenta un carácter tanto directo (están presentes directamente en la contabilidad de costes de las empresas) como indirecto, lo que no siempre da "visibilidad" al impacto global de determinados diseños. Es esta última relación indirecta la que impide a muchas empresas comprender el impacto de ciertas decisiones de diseño de envases y embalajes sobre los costes relacionados. Más aún, en muchas empresas ni siquiera existen datos $\mathrm{y} / \mathrm{o}$ información fiable que hagan visibles o transparentes total o parcialmente estos costes.

No obstante, cuanto intentamos medir la idoneidad de una determinada alternativa de envase y/o embalaje desde otras perspectivas diferentes a los costes, el sistema de evaluación o medición ya no resulta tan evidente $y$, en muchos casos, es muy difícil traducirlo completamente a términos económicos. Por ejemplo, el cumplimiento de la función protectora de los envases y embalajes podría medirse parcialmente en términos económicos con el coste de las pérdidas, deterioros 
o devoluciones. Sin embargo, resultaría más difícil medir la insatisfacción que estos problemas generan en cada parte de la cadena y, particularmente, en el cliente final, dado que no necesariamente se reflejan en las ventas (al menos, inmediatamente).

Una dificultad similar aparece cuando se quiere medir el impacto medioambiental de las diferentes alternativas de envase y embalaje, dado que si bien alguno de estos impactos lo podemos medir parcialmente en costes (por ejemplo, costes de gestión de residuos mediante la tasa del Punto Verde), parte de ellos se miden con una escala diferente a la económica. Este es el caso del sistema más empleado para medir el impacto medioambiental, la técnica ACV ("Análisis de Ciclo de Vida") o LCA ("Life Cycle Assessment" (ISO 14040; 2006). Esta técnica permite medir la huella de carbono generada no sólo por los envases y embalajes sino, de forma más global, por los productos a lo largo de su ciclo de vida (Williams et al., 2008; Levi et al., 2011; Williams y Wikström, 2011; Albretch et al., 2013; Wever y Voghtländer, 2013; Molina-Besch y Palsson, 2014; Bertolluci et al., 2014; Siracusa et al., 2014; Molina-Besch y Palsson, 2020).

Ante las dificultades para evaluar de forma objetiva cada alternativa de envase y embalaje desde una perspectiva multifuncional (no sólo la medioambiental o los costes, por ejemplo) y con un enfoque global de cadena se han desarrollado diferentes modelos de evaluación que combinan escalas cuantitativas con escalas cualitativas, lo cual, sin embargo, introduce cierta subjetividad en los resultados de estos análisis. El más extendido de estos últimos modelos es el método "Packaging Scorecard" (Olsmats y Dominic, 2003) que ha popularizado empresas como IKEA o Walmart. A partir del análisis de casos de cadenas de suministro y de envases y embalajes concretos establecen un sistema de valoración de alternativas combinando las necesidades de cada etapa en la cadena de suministro con criterios específicos cualitativos y cuantitativos.

\subsection{Modelizando el enfoque "Sustainable Packaging Logistics"}

En el contexto de los requisitos, los sistemas de valoración y las consideraciones organizativas expuestos anteriormente, surge la necesidad de conceptualizar la profunda relación entre el proceso de diseño del envase y embalaje, el diseño del producto y el diseño del sistema de la cadena de suministro (o sistema logístico). Este es el escenario perfecto para desarrollar un nuevo enfoque de diseño y gestión denominado "Packaging Logistics".

El término como tal comienza a acuñarse a finales de los 90 en el norte de Europa, y fue planteado inicialmente como la relación estructurada entre el sistema logístico y el sistema de envase y embalaje que permitiría mejorar los beneficios a las empresas. Este concepto se fue desarrollando y matizando posteriormente en su concepto más académico a partir de las aportaciones de diferentes autores, especialmente, procedentes de la Universidad sueca de Lund (Dominic et al., 2000; Saghir, 2002; Hellström y Saghir, 2007; Hellström y Nilsson, 2011; Pålsson y Hellström, 2016; Pålsson, 2018).

En el año 2002, Saghir propone una definición integrada de este concepto que ya enfatiza el rol activo del sistema de envase y embalaje para integrar diferentes visiones a nivel comercial, logístico y medioambiental y para afectar al sistema de producto y a la propia cadena de suministro y, todo ello, orientado a la mejora de la competitividad de las empresas.

Posteriormente, García-Arca et al. (2014) ajustan, matizan y amplían el concepto "Packaging Logistics" propuesto por Saghir (2002) al contexto de sostenibilidad, acuñando el término "Sustainable Packaging Logistics" (SPL) que definen como: “...el proceso de diseñar, implantar y controlar el sistema combinado de envase, embalaje y producto para una gestión segura, eficaz y eficiente de los flujos logísticos de materiales e información a lo largo de toda la cadena (incluyendo tanto las actividades de producción, manipulación, almacenamiento, transporte, venta y consumo, como las de recuperación, reutilización y/o eliminación de los residuos generados), con objeto de maximizar el valor para el consumidor y la sociedad, las ventas y los beneficios empresariales desde una perspectiva de sostenibilidad y en una dinámica de mejora continua”.

En esta definición hay una mención expresa a los procesos involucrados, al ámbito sostenible (social, económico y medioambiental) y a la visión dinámica de las cinco decisiones de diseño asociados al envase y embalaje. Tal como se ha comentado, esta visión dinámica es crítica para poder adaptar o matizar los requisitos de diseño a las amenazas y oportunidades del entorno. Asimismo, en esta última definición se refuerza la idea de integración de los tres sistemas involucrados: el envase y el embalaje, el producto y la propia cadena. Finalmente, se potencia la idea de conectar el diseño de envases y embalajes con la mejora competitiva de la empresa, esto es, la obtención de mejores resultados empresariales.

En este contexto, aprovechando las aportaciones previas descritas por García-Arca et al. (2017) y con la propia visión de los autores, tres serían los pilares que fundamentarían el despliegue del enfoque "Sustainable Packaging Logistics" en su perspectiva más amplia, esto es, contribuyendo al despliegue de la sostenibilidad en sus tres ámbitos (económico, social y medioambiental): 
- La definición de los requisitos de diseño, basada en la identificación de las necesidades globales de las diferentes etapas de la cadena de suministro desde una perspectiva sostenible. El nivel competitivo actual en la mayor parte de los mercados recomendaría la integración de todos estos requisitos sin despreciar ninguno, intentando lograr un consenso objetivo que satisfaga todas las prioridades de áreas y empresas a lo largo la cadena de suministro.

- La definición de un sistema que permita medir el impacto de determinadas decisiones de diseño (materiales, tecnologías, estructura de niveles, dimensiones y estética) sobre la eficiencia y sostenibilidad de la cadena de suministro. Este pilar está relacionado con la necesidad de medir y comparar los costes, el impacto medioambiental y cualquier otra variable asociada previamente comentada.

- La definición de una estructura organizativa que coordine todas las áreas afectadas por el diseño de los envases y embalajes a lo largo de la cadena de suministro, tanto internamente en cada empresa, como externamente en toda la cadena de suministro.

Se trataría, asimismo, de integrar el diseño del envase y el embalaje en las primeras fases de diseño del producto y de su sistema logístico y con un enfoque "dinámico" para adaptarse, tanto a las diferentes visiones y percepciones de los requisitos de diseño que, en cada momento, valora y percibe cada etapa de la cadena de suministro, como para adaptarse a los cambios que, en cada momento, se puedan producir en los propios requisitos de diseño y/o en el entorno. En la práctica, esto supondría que empresas y cadenas de suministro aprenden y se adaptan de forma eficiente y sostenible en materia de envases y embalajes, comportándose como organizaciones que aprenden ("learning organizations").

Por otro lado, para los autores el despliegue de estos tres pilares debería promover en las empresas una cultura del cambio, la innovación y la mejora continua basada en los envases y embalajes. Así, en el ámbito de los cambios e innovaciones se encontrarían las líneas de mejora ya adoptadas con éxito en otras empresas y cadenas de suministro pero que no pueden aplicarse indiscriminadamente sin conocer previamente los condicionantes de producto y de otros requisitos de diseño y de la propia cadena de suministro (para valorar esta idoneidad en cada caso es muy interesante la adopción sistemas de medición o evaluación de alternativas).

Lógicamente, en este campo de innovaciones se encontrarían las aportadas por la dinámica industria del envase y embalaje (por ejemplo, a nivel de materiales, calidades, y equipos-procesos de envasado o embalado). Así, la literarura científica proporciona algunos de estas líneas de mejora (García-Arca y Prado-Prado, 2008; Hellström y Nilsson, 2011; Kye et al., 2013; Garcia-Arca et al., 2017; Hellström y Olsson, 2017), que se enriquecen con las aportaciones de otros documentos o páginas web de carácter empresarial como ECR (www.ecr-community. org), EUROPEN (www.europen-packaging.eu), AECOC (www.aecoc.es) y ECOEMBES (www.ecoembes.com). En la tabla 2 se presentan un resumen de estos posibles cambios o innovaciones, indicando el potencial impacto en los costes globales a nivel de sostenibilidad. 
Tabla 2 Relación de potenciales actuaciones para mejorar los envases y embalajes.

\begin{tabular}{|c|c|c|c|}
\hline Línea de mejora & $\begin{array}{c}\text { Beneficios potenciales en la } \\
\text { sostenibilidad económica } \\
\text { (reducción) }\end{array}$ & $\begin{array}{c}\text { Beneficios potenciales en } \\
\text { sostenibilidad medioambiental } \\
\text { (reducción) }\end{array}$ & $\begin{array}{l}\text { Beneficios potenciales } \\
\text { sostenibilidad social }\end{array}$ \\
\hline $\begin{array}{l}\text { Cambios dimensiones en } \\
\text { envases y/o embalajes (a } \\
\text { nivel de envase primario, } \\
\text { secundario y/o terciario) }\end{array}$ & $\begin{array}{l}\text { Costes de compra de envases y } \\
\text { embalajes } \\
\text { Coste logístico (manipulación, } \\
\text { almacenamiento y transporte) } \\
\text { Costes de gestión de residuo }\end{array}$ & $\begin{array}{l}\text { Consumo de materias primas y } \\
\text { generación de residuos } \\
\text { Consumo energético } \\
\text { y contaminación } \\
\text { (transporte) }\end{array}$ & 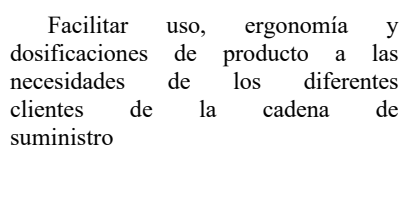 \\
\hline $\begin{array}{l}\text { Cambio en los materiales } \\
\text { empleados en los envases } \\
\text { y/o embalajes (tipo material, } \\
\text { calidad...) }\end{array}$ & $\begin{array}{l}\text { Costes de compra de envases y } \\
\text { embalajes } \\
\text { Costes gestión residuo }\end{array}$ & $\begin{array}{l}\text { Reciclabilidad } \\
\text { Capacidad de valorización } \\
\begin{array}{l}\text { Consumo de materias primas, } \\
\text { energía y generación de } \\
\text { residuos (según material y } \\
\text { calidad) }\end{array} \\
\begin{array}{l}\text { Consumo energético y } \\
\text { contaminación (transporte) }\end{array}\end{array}$ & $\begin{array}{l}\text { Facilitar uso } \\
\text { Percepción de calidad de la } \\
\text { calidad del producto }\end{array}$ \\
\hline $\begin{array}{lllr}\text { Variación } & \text { de } & \text { la } & \text { cantidad de } \\
\text { producto } & \text { por } & & \text { envase } \\
\text { primario } & \text { o } & \text { en } & \text { la } \\
\text { forma/tamaño del producto } & \end{array}$ & $\begin{array}{l}\begin{array}{l}\text { Coste de compra de envases y } \\
\text { embalajes (por unidad de } \\
\text { producto) }\end{array} \\
\begin{array}{l}\text { Coste logístico (manipulación, } \\
\text { almacenamiento y transporte) } \\
\text { (por unidad producto) }\end{array} \\
\text { Costes de gestión de residuo } \\
\text { (por unidad de producto) }\end{array}$ & 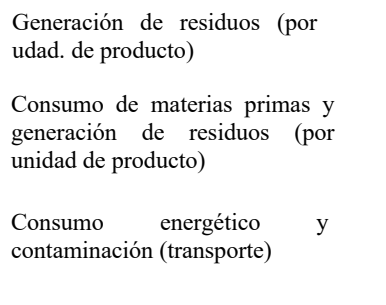 & $\begin{array}{l}\text { Facilitar uso, ergonomía y } \\
\text { dosificaciones de producto a las } \\
\text { necesidades de los diferentes } \\
\begin{array}{l}\text { clientes de la cadena de } \\
\text { suministro }\end{array} \\
\text { Percepción calidad del producto }\end{array}$ \\
\hline $\begin{array}{lrr}\text { Cambio en la forma de } \\
\text { envasar y/o embalar } & \text { (nuevos } \\
\text { equipos, nuevos } & \text { útiles, } \\
\text { nuevas tecnologías,...) }\end{array}$ & $\begin{array}{l}\text { Coste productivo de envasado y/ } \\
\text { o embalado } \\
\begin{array}{l}\text { Costes de deterioros o } \\
\text { rechazos }\end{array} \\
\text { Costes de gestión de residuos }\end{array}$ & $\begin{array}{l}\begin{array}{l}\text { Generación de residuos } \\
\text { producción; }\end{array} \text { (en } \\
\text { nueva tecnología de envasado } \\
\text { puede alargar la vida útil del } \\
\begin{array}{l}\text { producto, disminuyendo } \\
\text { obsoletos) }\end{array} \\
\begin{array}{l}\text { los } \\
\text { Consumo energético en }\end{array} \\
\begin{array}{l}\text { procesos los } \\
\text { embalado }\end{array}\end{array}$ & $\begin{array}{l}\text { Facilitar uso y alargamiento de la vida } \\
\text { útil del producto } \\
\begin{array}{l}\text { Percepción de la calidad del } \\
\text { producto }\end{array}\end{array}$ \\
\hline $\begin{array}{lrrrr}\begin{array}{l}\text { Cambio } \\
\text { envases }\end{array} & \text { en } & \text { el } & \text { número } & \text { de } \\
\text { (número } & \text { de } & & \text { embalaje } \\
\text { primarios } & \text { por } & \text { cada } & \text { envases } \\
\text { envase } & \\
\text { secundario) } & & & \end{array}$ & $\begin{array}{l}\begin{array}{l}\text { Coste de compra de envases y } \\
\text { embalajes (por unidad de } \\
\text { producto) }\end{array} \\
\begin{array}{l}\text { Coste logístico } \\
\text { almacenamiento y }\end{array} \text { (manipulación, } \\
\text { (por unidad de producto) } \\
\begin{array}{l}\text { (incluye manipulación en el } \\
\text { punto de venta) }\end{array} \\
\text { Costes de gestión de residuo } \\
\text { (por unidad de producto) }\end{array}$ & 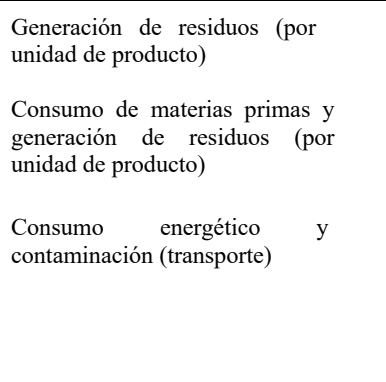 & \begin{tabular}{lllr} 
Facilitar uso, ergonomía & \multicolumn{2}{c}{ y } \\
dosificaciones de & producto a las \\
necesidades de los diferentes \\
clientes de la cadena de \\
suministro
\end{tabular} \\
\hline $\begin{array}{l}\text { Estandarización } \\
\text { dimensiones, formatos } \\
\text { los envases y/o embalajes }\end{array}$ & $\begin{array}{l}\text { Coste de compra de envases y } \\
\text { embalajes (por economías de } \\
\text { escala) } \\
\text { Coste productivo (reducción de los } \\
\text { tiempos de preparación) } \\
\text { Costes de gestión de residuo }\end{array}$ & 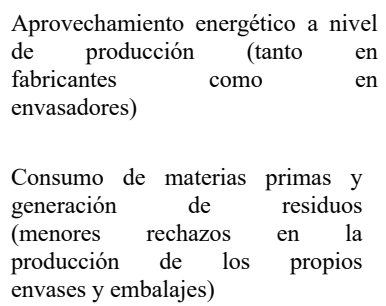 & $\begin{array}{l}\begin{array}{l}\text { Mejor comunicación con } \\
\text { cliente ("reconocimiento }\end{array} \text { de } \\
\text { familia del producto } \\
\text { proveedor") }\end{array}$ \\
\hline
\end{tabular}




\begin{tabular}{|c|c|c|c|}
\hline & & $\begin{array}{l}\text { Consumo energético } \\
\text { contaminación (transporte) }\end{array}$ & \\
\hline $\begin{array}{l}\text { Estandarización } \\
\text { calidades en los materiales } \\
\text { empleados en los envases y/o } \\
\text { embalajes }\end{array}$ & 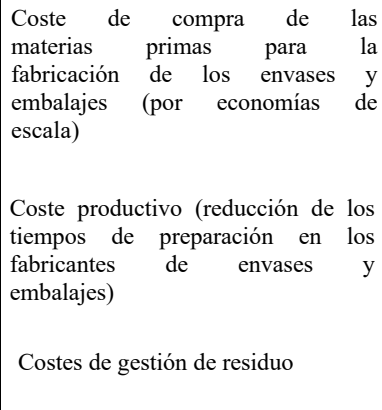 & $\begin{array}{l}\begin{array}{l}\text { Aprovechamiento } \\
\text { nivel } \\
\text { (fabricantes de }\end{array} \text { de } \\
\text { embalajes) }\end{array}$ & 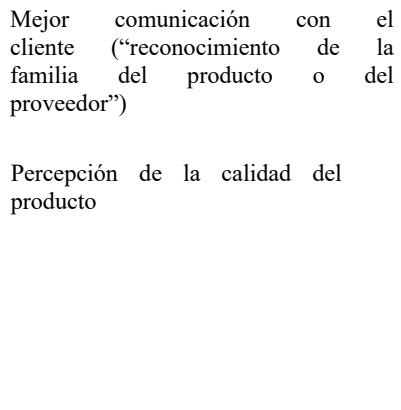 \\
\hline $\begin{array}{l}\text { Eliminación } \\
\text { "sobreembalajes" }\end{array}$ & $\begin{array}{l}\text { Coste de compra de envases y } \\
\text { embalajes } \\
\text { Coste logístico (mejor } \\
\text { aprovechamiento del volumen) } \\
\text { Costes gestión residuo }\end{array}$ & $\begin{array}{l}\text { Consumo de materias primas y } \\
\text { generación de residuos } \\
\begin{array}{l}\text { Consumo energético y } \\
\text { contaminación (transporte) }\end{array}\end{array}$ & $\begin{array}{l}\text { Facilitar uso } \\
\text { Percepción de la calidad del } \\
\text { producto }\end{array}$ \\
\hline $\begin{array}{l}\text { Cambio en el diseño del Arte } \\
\text { Gráfica o estética del envase y/o } \\
\text { embalaje (colores, } \\
\text { textos, etiquetas, formas...) }\end{array}$ & $\begin{array}{l}\text { Coste de compra de envases y } \\
\text { embalajes }\end{array}$ & \begin{tabular}{llrr} 
Consumo & de & materias primas, \\
energía & y & \multicolumn{2}{c}{ generación de } \\
residuos & \multicolumn{2}{c}{ (simplificación } \\
productiva; estandarización de \\
textos multilingües)
\end{tabular} & $\begin{array}{l}\text { Facilitar uso } \\
\text { Mejor comunicación con el } \\
\text { cliente ("visibilidad") Percepción } \\
\text { calidad producto }\end{array}$ \\
\hline $\begin{array}{l}\text { Implantación de embalajes } \\
\text { reutilizables }\end{array}$ & $\begin{array}{l}\text { Coste de compra de envases y } \\
\text { embalajes } \\
\begin{array}{l}\text { Coste logístico (manipulación, } \\
\text { almacenamiento y transporte) } \\
\text { (incluye logística inversa) }\end{array} \\
\text { Costes de gestión de residuo }\end{array}$ & $\begin{array}{l}\text { Consumo de materias primas y } \\
\text { generación de residuos } \\
\begin{array}{l}\text { Consumo energético y } \\
\text { contaminación (transporte) }\end{array}\end{array}$ & $\begin{array}{l}\text { Facilitar uso } \\
\text { Percepción de la calidad del } \\
\text { producto }\end{array}$ \\
\hline 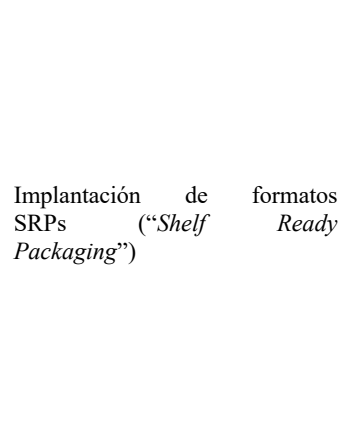 & $\begin{array}{l}\begin{array}{l}\text { Coste de compra de envases y } \\
\text { embalajes (por unidad de } \\
\text { producto) }\end{array} \\
\text { Coste productivo de envasado y } \\
\text { embalado } \\
\text { Coste logístico (manipulación, } \\
\text { almacenamiento y transporte) (por } \\
\text { unidad de producto) (incluye } \\
\text { manipulación en el punto de } \\
\text { venta) a }\end{array}$ & $\begin{array}{l}\text { Consumo de materias primas y } \\
\text { generación de residuos (por } \\
\text { unidad de producto) } \\
\begin{array}{l}\text { Consumo energético y } \\
\text { contaminación (transporte) }\end{array}\end{array}$ & $\begin{array}{l}\text { Facilitar uso } \\
\begin{array}{l}\text { Mejor comunicación con el } \\
\text { cliente ("visibilidad") }\end{array} \\
\begin{array}{l}\text { Percepción de la calidad del } \\
\text { producto }\end{array}\end{array}$ \\
\hline
\end{tabular}




\section{Objetivos y metodología del estudio}

En el marco conceptual planteado anteriormente, la principal cuestión aplicada que intenta dilucidar este artículo es explorar si el desarrollo de un modelo estructurado para el despliegue del SPL puede contribuir activamente a la promoción de cambios e innovaciones que contribuyan a la mejora de la sostenibilidad (económica, medioambiental y social) de empresas y cadenas de suministro.
Así, los autores esquematizan en la figura 1 el modelo de gestión asociado al despliegue del "Sustainable Packaging Logistics". Este esquema refleja la importancia de reforzar los tres pilares para generar una cultura de cambio e innovación que proporcione activamente una mejora global del comportamiento sostenible de empresas y cadenas de suministro, a nivel económico, medioambiental y social. Todo ello integrado, además, en el propio diseño del producto y de la cadena de suministro.
Figura 1 Modelo de despliegue del "Sustainable Packaging Logistics" desde una perspectiva sostenible

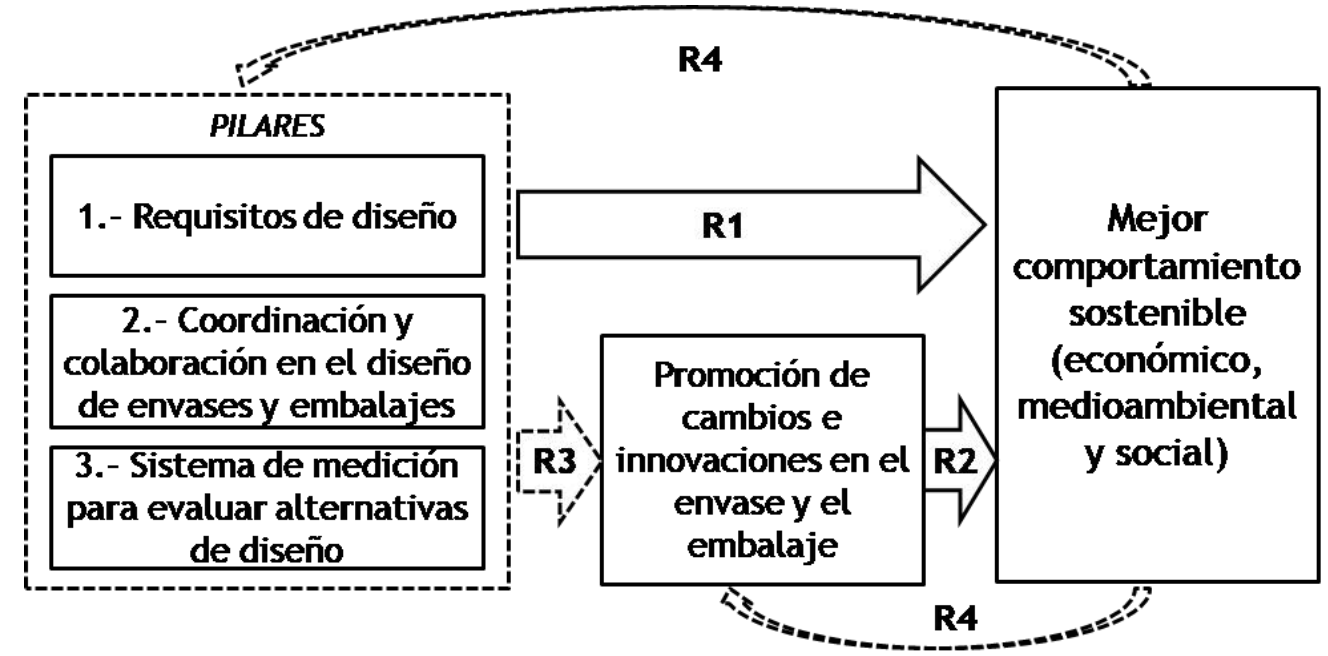

- Hipótesis 2: Cuanto mayor es el nivel de coordinación y colaboración interna en el proceso de diseño, mejor es el resultado sostenible. Para llegar a probar esta hipótesis se pregunta a las empresas no sólo sobre el propio nivel de coordinación y colaboración interna entre diferentes áreas y departamentos en el diseño del envase y el embalaje, sino también por el nivel de integración de este diseño con el del propio producto $(\mathrm{R} 1$, pilar 2).

Por otro lado, el despliegue de los tres pilares, también podría contribuir a una mayor promoción de cambios e innovaciones que son los que después, directamente, podrían impactar en unos mejores resultados. En el estudio descrito en este artículo, se explora las relaciones directas indicadas en la figura 1 (R1 y R2), dejando las otras indirectas (R3 y R4) para posteriores estudios. En este contexto, las hipótesis que se intentan validar son las siguientes:

- Hipótesis 1: Cuanta mayor es la importancia otorgada a un requisito en el proceso de diseño, mejor es el resultado sostenible. Para llegar a probar esta hipótesis se pregunta a las empresas cuál es el nivel de importancia que se otorga a cada uno de los requisitos de los nueve identificados en la literatura (tabla 1; comerciales, protectores, productivos, logísticos, compras, medioambientales, ergonómicos, legales y comunicación) (R1, pilar 1).
- Hipótesis 3: Cuanto mayor es el nivel de coordinación y colaboración externa en el proceso de diseño, mejor es el resultado sostenible. Para llegar a probar esta hipótesis se pregunta a las empresas sobre tres cuestiones: por un lado, sobre el nivel de coordinación en el proceso de diseño con los proveedores de envases y embalajes; por otro, sobre el nivel de coordinación en el proceso de diseño con distribuidores; finalmente, sobre el nivel de coordinación interno en el proceso de diseño de las unidades de carga y embalajes en los aprovisionamientos procedentes de los proveedores de materias primas y componentes (R1, pilar 2). 
- Hipótesis 4: Cuanto mayor es el desarrollo de sistemas de medición de alternativas de envases y embalajes, mejor es el resultado sostenible. Para llegar a probar esta hipótesis se pregunta a las empresas sobre ocho sistemas diferentes: cinco basados en costes (compras, producción, logística interna, logística externa y gestión residuos) y tres basado en otros criterios (Packaging Scorecard, Análisis del Ciclo de Vida y Benchmarking) (R1, pilar 3).

- Hipótesis 5: Cuanta mayor es la predisposición al cambio, la mejora y a la innnovación en relación con los envases y embalajes, mejor es el resultado sostenible. Para llegar a probar esta hipótesis se pregunta a las empresas sobre la frecuencia o importancia de los cambios e innovaciones en este campo (de los 11 identificados en la literatura; tabla 2) (R2).

Para desarrollar el estudio se profundiza en la relación que existe entre un mayor despliegue de los aspectos considerados en el modelo con la obtención de un mejor resultado en sostenibilidad, empleando como referencia lo analizado en una muestra de empresas fabricantes pertenecientes al sector de menaje en España.

La recopilación de información para el estudio se basa en el cuestionario electrónico con preguntas cerradas que emplean como referencia la escala 1-5 (uno valoración mínima; 5 valoración máxima), basada en la propuesta por Likert (1932), para medir la importancia, la integración y/o el impacto manifestado por las empresas en cada uno de las preguntas del cuestionario que estaban asociadas a los aspectos del modelo anteriormente comentados, así como a los resultados sostenibles obtenidos. Cada una de las empresas del sector fue contactada por teléfono, para presentarle el estudio y solicitar su participación (los principales puestos contactados estaban relacionados con la responsabilidad productiva o logística). A las empresas que respondían afirmativamente, se les enviaba un correo electrónico con un enlace individualizado donde podían contestar al cuestionario. El número final de cuestionarios válidos fue de 66. El período de recepción de estos cuestionarios se extendió durante 3 meses.

En el cuestionario también se incluían aspectos vinculados a los resultados sostenibles alcanzados gracias al diseño (o rediseño) de envases y embalajes. Concretamente, se les pedía a las empresas que valorasen con una escala Likert (1-5) la importancia o impacto en los siguientes aspectos vinculados a la sostenibilidad: la mejora en la capacidad de diferenciación y el beneficio social del producto, la mejora en la capacidad protectora del producto (para evitar deterioros y roturas), la reducción de los costes totales (a nivel interno y externo en toda su cadena de suministro) y la mejora en el comportamiento medioambiental (de nuevo, a nivel interno y externo en toda su cadena de suministro).
Para realizar el análisis, se han establecido dos grupos de empresas en función de los mejores (G1) o peores (G2) resultados alcanzados con el diseño de los envases y embalajes a nivel de sostenibilidad. Estos resultados cuantitativos se obtenían como suma de las valoraciones de los aspectos comentados anteriormente. En cada grupo se ubicaban 33 empresas. Las diferencias globales entre las medias de los resultados entre ambos grupos eran estadísticamente significativas ("p-value" $=2.792 \mathrm{E}-12$ ).

Para comparar y justificar el potencial de cada cuestión analizada se ha realizado un test de Mann-Whitney. El test no paramétrico de Mann-Whitney hace posible la comprobación de las medias de dos grupos para ver si éstas son iguales o diferentes estadísticamente. Normalmente, las diferencias entre dos grupos se consideran significativas cuando el "p-value" está por debajo de $0.05\left(^{*}\right)$, o de forma más exigente, cuando este "p-value" está por debajo de 0.01 $\left({ }^{* *}\right)$. En este sentido, la muestra de empresas considerada (66) se puede considerar suficiente para aplicar este test, si bien, cuando el contraste indique que es no significativo ("p-value" >0.05) esto no implique que las hipotésis puedan ser rechazadas.

\section{Resultados del estudio}

Con el objeto de presentar los resultados del análisis empírico en el sector del menaje, este apartado se divide inicialmente en cuatro grandes bloques, coincidentes con cada uno de los tres pilares del modelo, junto con un bloque dedicado a la descripción de los resultados en el campo de los cambios e innovaciones asociados a los envases y embalajes. Asimismo, a estos cuatro bloques se les añade un último epígrafe donde se incluyen unas valoraciones y reflexiones globales.

\subsection{Análisis de los requisitos de diseño}

Los resultados del análisis en este primer pilar del modelo muestran resultados positivos en el grupo de empresas con mejores resultados (G1), al otorgar más importancia a 4 (legales, comunicación, medioambientales y ergonómicos) de los 9 requisitos considerados (ver figura 2).

El análisis no encuentra, sin embargo, diferencias significativas en cinco de los requisitos más mencionados en la literatura académica (protectivos, logísticos, comerciales, compras y productivos). Entre estos últimos requisitos de diseño se encuentran los de más importancia para las empresas de ambos grupos; la excepción a esta afirmación lo constituyen los requisitos de tipo legal.

Curiosamente, las diferencias significativas se encuentran entre los requisitos con menor importancia para las empresas (salvo, los ya mencionados de tipo legal). La situación 
anterior parece apuntar a que las empresas con peores resultados presentan una menor comprensión del carácter multifacético del envase y embalaje que hace que otorgue una menor importancia a algunos aspectos que completan, junto otros aspectos sí valorados por todas las empresas, esta visión holística del diseño.

Figura 2 Analísis de

importancia de los

- Media G1 (Mejores Resultados

requisitos de diseño entre

los dos grupos de empresas

(entre paréntesis, "p-value")

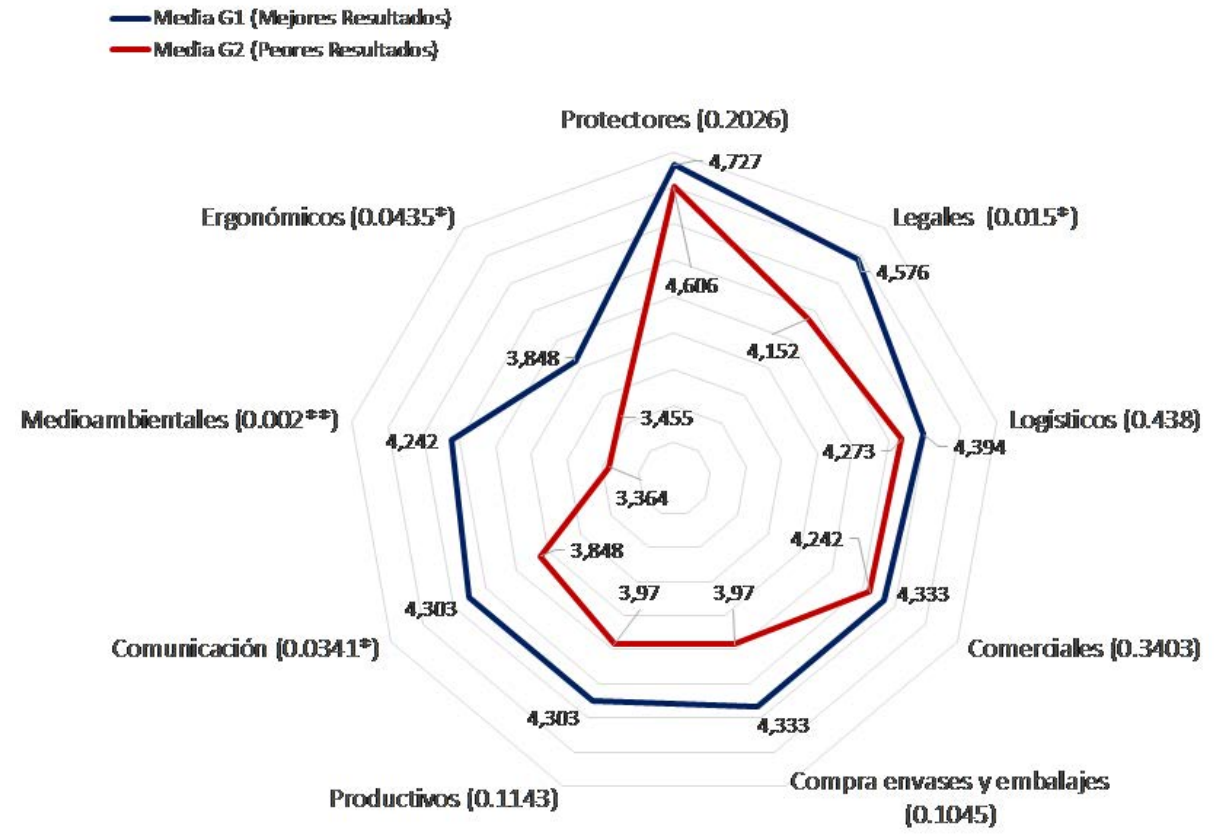

\section{Análisis de la coordinación y colaboración en el diseño de envases y embalajes}

Relacionado con lo comentado anteriormente, podría plantearse que una mayor sensibilidad hacia una visión más completa de los requisitos de diseño podría estar íntimamente relacionada con una mayor coordinación y colaboración a lo largo de la cadena de suministro. Dado que cada empresa y departamento dentro de esta cadena de suministro puede tener una visión diferente en cuanto a la importancia relativa de cada requisito, parece razonable que en el proceso de diseño se potenciase la colaboración y coordinación (interna y externamente) entre todas las partes afectadas por el mismo.

Así, a nivel interno, se han encontrado diferencias significativas entre ambos grupos de empresas envasadoras, a nivel de una mayor coordinación e integración internas del proceso de diseño de envase y embalaje (ver figura 3); estas diferencias también se encuentran a nivel de la coordinación e integración del diseño de envases y embalajes con el diseño del producto. Este último aspecto podría relacionarse con el hecho, ya comentado, de que las empresas con mejores resultados presentan una visión más completa de los requisitos de diseño.
Por otro lado, el análisis realizado en este segundo pilar del modelo propuesto a nivel externo valida mayoritariamente esta suposición, por cuanto se encuentran diferencias significativas en dos de los tres aspectos considerados en su aportación positiva a los resultados sostenibles (ver figura 3). La excepción a la afirmación anterior se ubica aguas abajo en la cadena a nivel de distribuidores; la posible razón que explicaría este resultado podría asociarse a que, en el sector analizado, los distribuidores (mayoritariamente, pertenenecientes a la "gran distribución") son los que lideran la coordinación con las empresas envasadoras (en aspectos no sólo vinculados a envases, embalajes y unidades de carga) por lo que condicionaría por igual a todas las empresas, independientemente de su pertenencia a uno u otro grupo (G1 y G2).

No obstante, el proceso de coordinación en el diseño aguas arriba en la cadena (con proveedores de envases y embalajes) es liderado por las empresas envasadoras, por lo que las más avanzadas (G1) estarían más motivadas en promover la colaboración y la coordinación relacionadas con el diseño de sus envases y embalajes que redunden en un mejor resultado sostenible global. Asimismo, también se han encontrado diferencias significativas positivas en el nivel de coordinación entre las envasadoras y sus proveedores de materias primas y componentes (para promover embalajes y unidades de carga eficientes y sostenibles en los aprovisionamientos). 
Figura 3 Analisis del nivel de coordinación interna y externa en el diseño de envase y embalaje (entre paréntesis, "p-value")

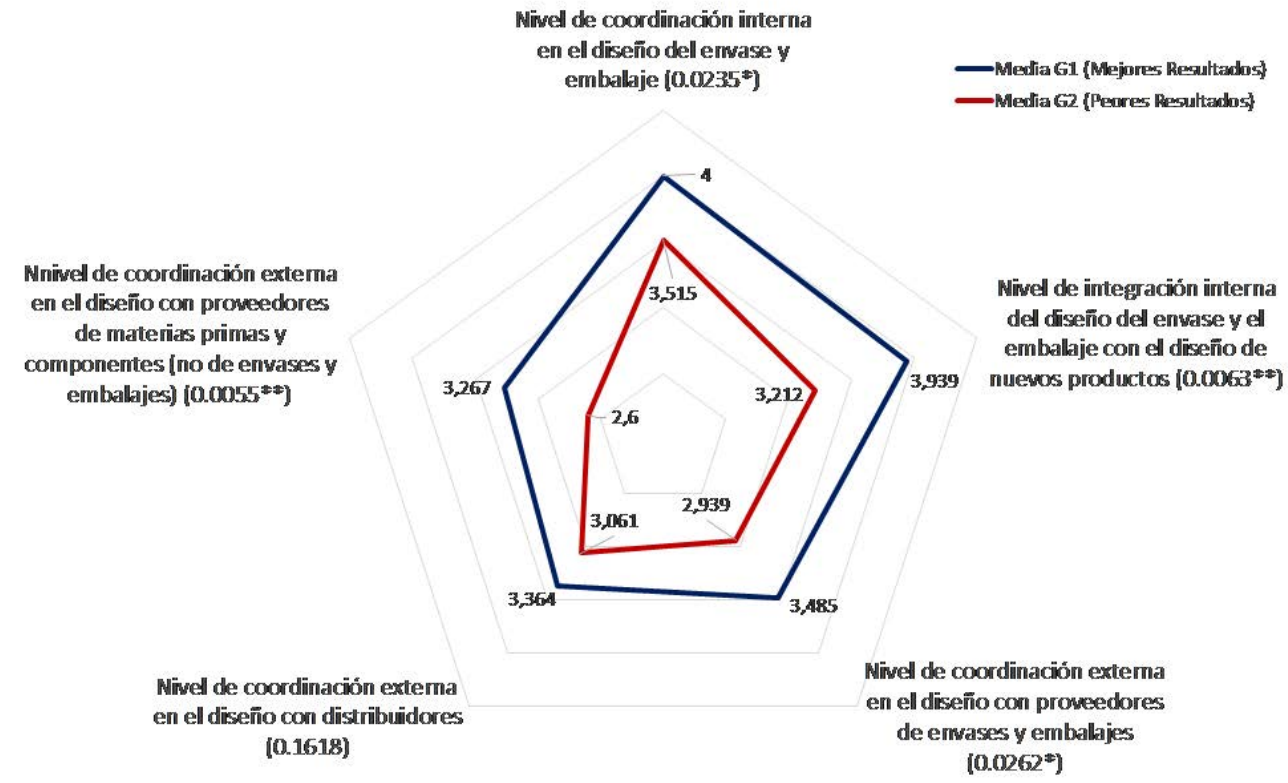

en los costes logísticos (internos y externos) es mayor en las empresas con mejores resultados (G1) que en las peores (G2) (ver figura 4). Sin embargo, no se han encontrado diferencias significativas en otros sistemas de costes como los de compra y los de gestión de residuos (en ambos casos, con un carácter más directo y visible $\mathrm{y}$, por tanto, más fáciles de implantar y conectar con las decisiones de diseño para todo tipo de empresas), ni con los costes de envasado y embalado (que suelen encuadrarse de forma más amplia en los sistemas de costes productivos que son también ampliamente desarrollados y conocidos por todo tipo de empresas).
En relación con el tercer pilar del modelo, el análisis muestra que el desarrollo de sistemas de medición basados 
Figura 4 Análisis de la implantación de sistemas de medición basados en diferentes tipos de costes (entre paréntesis, "p-value")

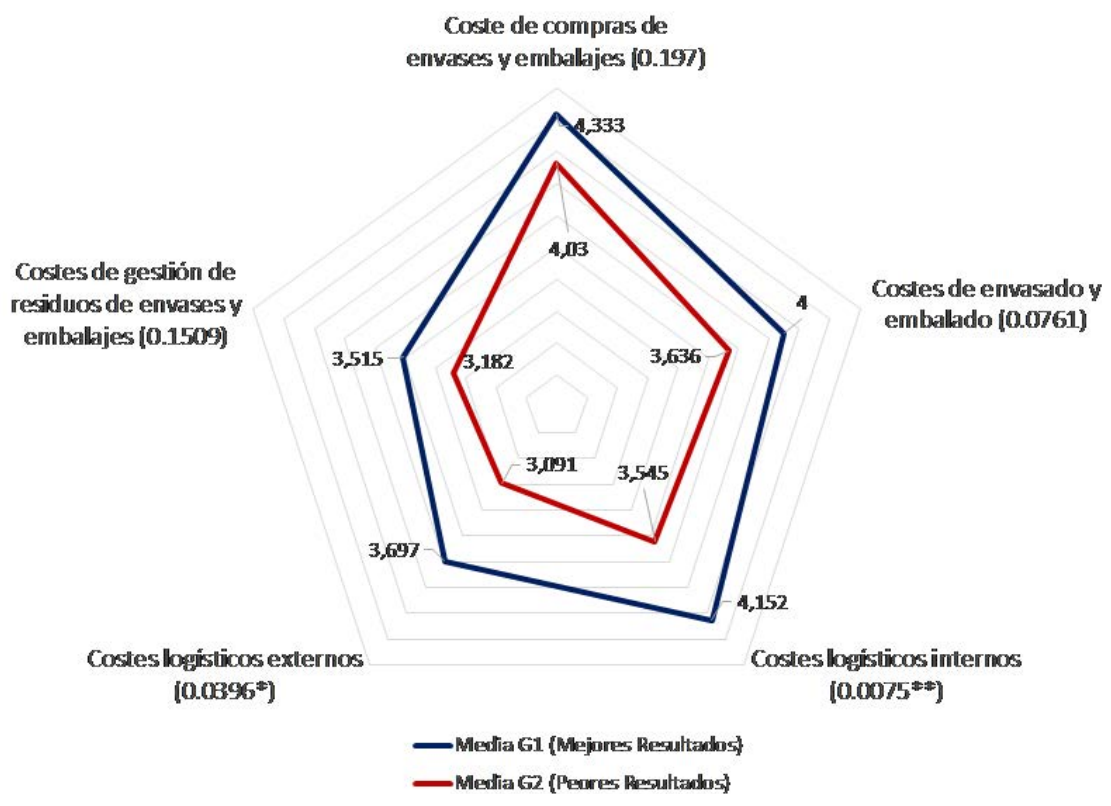

Por otro lado, estas diferencias a nivel de sistemas de costes logísticos también se vuelven a encontrar en el nivel de desarrollo de sistemas de medición basados en otros criterios (diferentes de los costes), particulamente, en sistemas basados en las técnicas "Packaging Scorecard" y Análisis de Ciclo de Vida (LCA) (ver figura 5). Sin embargo, no se han encontrado diferencias significativas entre los dos grupos de empresas a nivel de despliegue de sistemas de medición basados en técnicas "Benchmarking” (en la mayor parte de los casos, basadas en la comparación comercial de alternativas, a nivel interno o entre productos de los competidores), lo que sería coherente con la similar importancia otorgada a los requisitos de diseño de tipo comercial. 
Figura 5 Analisis del nivel de implantación de sistemas de medición no basados en costes (entre paréntesis, "p-value")

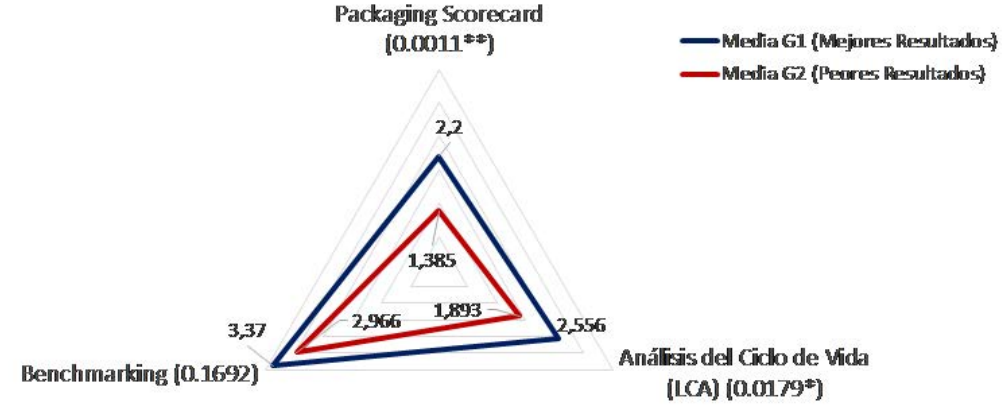

Así, se han encontrado diferencias significativas entre los dos grupos en 7 de los 11 tipos de acciones consideradas (ver figura 6), lo que implicaría que las empresas con mejores resultados presentan una mayor predisposición a la realización de cambios e innovaciones en sus envases y embalajes.

Buena parte de los cambios e innovaciones con diferencias significativas entre ambos grupos, aunque no todos, se encuadran en la órbita medioambiental (incluyendo, la reducción de sobreembalajes, los cambios de materiales, el cambio en el número de envases por embalaje, la implantación de SRPs ("Shelf Ready Packaging") y el uso de embalajes reutilizables). Todo ello es coherente con la mayor importancia otorgada por este grupo de empresas (G1) a los requisitos de diseño medioambientales y a la adopción de sistemas específicos de medición como el Ciclo de Vida del Producto. No obstante, otros cambios o innovaciones en los que también se han encontrado diferencias significativas tienen además un fuerte impacto en la eficiencia de la cadena de suministro a nivel productivo o logístico; este es el caso de acciones como la "estandarización dimensional" o "el cambio en la forma de envasar (o embalar) los productos".

Por otro lado, el hecho de que en los cambios de tipo más comercial ("cambios estéticos" y "cambios en la cantidad de producto por envase") no se hayan encontrado diferencias significativas es coherente con la similar importancia encontrada en los requisitos de diseño de tipo comercial y en el similar nivel de implantación de sistemas de medición con un especial enfoque comercial como el "benchmarking". Asimismo, tampoco se han encontrado diferencias significativas en otras dos categorías de cambios (cambios de dimensiones y estandarización de materiales) que, no obstante, también podrían estar relacionadas con otras categorías donde sí se han encontrado diferencias significativas entre ambos grupos de empresas.

Por ejemplo, si las empresas con mejores resultados promueven en mayor medida la estandarización dimensional en sus envases y embalajes, esta medida supone cambios

alcanzados en cuanto a la predisposición de las empresas para realizar cambios e innovaciones en sus envases y embalajes. 
en sus dimensiones; asimismo, si las empresas con mejores resultados promueven en mayor medida cambios en los materiales o en el proceso de envasado/embalado, indirectamente también podrían estar promoviendo cierta estandarización de materiales.

En la literatura académica y empresarial se pueden encontrar múltiples ejemplos de los potenciales beneficios de estos cambios. Buena parte de estos ejemplos se centran en grandes empresas (por ejemplo, Hellström y Nilsson, 2011;
Pålsson et al., 2013; García-Arca et al., 2014; Sohrabpour et al., 2016; Hellström y Olsson, 2017). Sin embargo, no es tan frecuente encontrar en la literatura ejemplo de estos cambios en pequeñas y medianas empresas, lo que sin duda plantea otro interesante punto de estudio en investigaciones futuras. Algunos ejemplos de cambios en pequeñas y medianas empresas se pueden encontrar en ECOEMBES (www. ecoembes.es), la organización que gestiona en España los aspectos vinculados al Punto Verde.

Figura 6 Análisis de la

intensidad de cambios

e innovaciones en los

envases y embalajes (entre

paréntesis, "p-value")

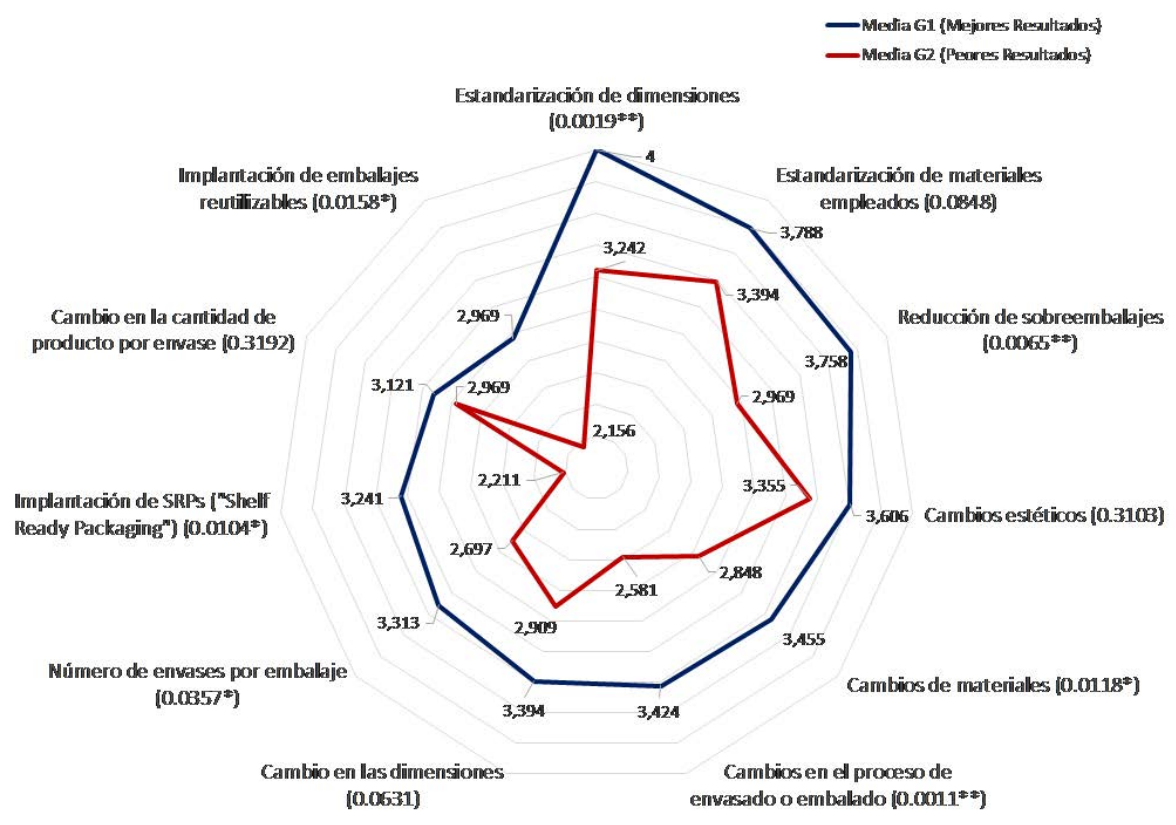

\subsection{Análisis general de los resultados del estudio en el sector de menaje}

El análisis del proceso de diseño de envases y embalajes en el sector de menaje presenta evidencias empíricas del interés de adoptar el modelo de despliegue del enfoque SPL como medio de contribución a la mejora de la sostenibilidad de las cadenas de suministro. Lógicamente, con toda probabilidad existen otros factores no contemplados en el proceso de diseño que impactan (positiva o negativamente) en el comportamiento sostenible de las cadenas de suministro, aunque los resultados obtenidos son prometedores e ilustrativos. En este sentido, el análisis de los resultados valida parcialmente las hipótesis de investigación inicialmente planteadas:

- Hipótesis 1: Cuanta mayor es la importancia otorgada a un requisito en el proceso de diseño, mejor es el resultado sostenible. Así, las empresas con mejores resultados son las que otorgan una mayor importancia en el diseño a 4 de los 9 requisitos identificados.

- Hipótesis 2: Cuanto mayor es el nivel de coordinación y colaboración interna en el proceso de diseño, mejor es el resultado sostenible. En este punto se han encontrado diferencias significativas, tanto en la coordinación interna para el diseño de envases y embalajes, como en la propia integración del proceso de diseño de envases y embalajes con el proceso de diseño del producto.

- Hipótesis 3: Cuanto mayor es el nivel de coordinación y colaboración externa en el proceso de diseño, mejor es el resultado sostenible. También en este punto se ha encontrado esta relación positiva en la coordinación y colaboración en diseño con proveedores (de envases y embalajes, pero también de materias y componentes para las empresas envasadoras), aunque no con 
cdistribuidores. En cualquier caso, el rol que los distribuidores juegan liderando el proceso de creación de algunos productos (y de sus envases y embalajes asociados) es un punto de interés en futuras investigaciones, dado que en el estudio actual sólo se recoge la perspectiva de las empresas envasadoras.

- Hipótesis 4: Cuanto mayor es el desarrollo de sistemas de medición de alternativas de envases y embalajes, mejor es el resultado sostenible. En este sentido, las empresas con mejores resultados presentan un mayor desarrollo de sistemas de medición a nivel de costes logísticos (internos y externos), pero también a nivel de algunas técnicas que evalúan otros criterios (el Análisis del Ciclo de Vida y el método Packaging Scorecard).

- Hipótesis 5: Cuanta mayor es la predisposición al cambio, la mejora y a la innnovación en relación con los envases y embalajes, mejor es el resultado sostenible. El análisis muestra un impacto positivo en esta cuestión dado que existen diferencias significativas en 7 de los 11 cambios o innovaciones considerados, particularmente, los que presentan un mayor impacto a nivel medioambiental (reducción de residuos y/o reducción de materias primas empleadas).

En el contexto anterior, la aportación más relevante del estudio realizado en el sector de menaje es que una mayor estructuración del proceso de diseño de envases y embalajes (el modelo propuesto) contribuiría activamente a la mejora de los resultados sostenibles en las empresas y cadenas de suministro. Sin embargo, el estudio no analiza ni muestra los estados por los que han pasado las empresas para llegar a la situación actual. Este análisis evolutivo podría explicar, con un mayor nivel de detalle, las motivaciones y dificultades que las empresas han encontrado para desarrollar algunos de los aspectos del modelo, lo que también supone otra perspectiva diferente que se debería abordar en investigaciones futuras.

Las principales limitaciones del artículo están asociadas a la subjetividad de las valoraciones realizadas por las empresas y que estas valoraciones representan sólo la perspectiva de una parte de la cadena de suministro (las empresas envasadoras). Además, el estudio analiza un número limitado de empresas (66), concentrándose éstas en un único sector (menaje y productos del hogar). Por otro lado, el perfil de la persona entrevistada en cada empresa también puede presentar algún sesgo hacia las consideraciones sostenibles de tipo productivo y logístico en detrimento de otros enfoques también muy importantes como el punto de vista comercial.

Asimismo, el estudio se centra en explorar las relaciones directas cuantitativas R1 y R2 del modelo propuesto (y no las indirectas R3 y R4; ver figura 1). Por otro lado, para desarrollar el análisis los autores han incluido en el grupo de empresas con mejores resultados (G1) a aquellas empresas con un valor más alto en la suma de las valoraciones de los aspectos considerados en dichos resultados y no de forma independiente, lo que podría matizar la asignación de alguna empresa a uno u otro grupo. Todas estas limitaciones serán contempladas en investigaciones futuras por parte de los autores.

En cualquier caso, los autores consideran que el enfoque aplicado y el modelo propuesto pueden ser de interés, tanto para el mundo académico como el empresarial. Los contenidos presentados pueden ayudar a mejorar la comprensión y conocimiento de cómo funciona la relación producto-envase-embalaje-cadena de suministro con objeto de mejorar la sostenibilidad. Así, los investigadores cuentan con un modelo conceptual que proporciona nuevas líneas de estudio para profundizar en la relación existente entre la estructuración del proceso de diseño y la obtención de mejores resultados sostenibles. Por otro lado, las empresas pueden disponer de un amplio abanico de recomendaciones y líneas de trabajo para orientar sus estrategias y políticas asociadas al proceso de diseño de envases y embalajes que redunden en un mejor comportamiento sostenible.

De hecho, el interés de estos planteamientos para el mundo académico y empresarial es otro incentivo más para promover una mayor colaboración conjunta entre investigadores y organizaciones en este campo, en línea con las metodologías basadas en el "estudio de casos" y en "Action Research" (Näslund et al., 2010).

\section{Conclusiones}

El envase y el embalaje es un aspecto clave para entender el diseño y la gestión de una cadena de suministro sostenible, por cuanto reproduce a una escala operacional las diferentes perspectivas y necesidades de cada empresa en la búsqueda de un funcionamiento eficiente y coordinado. Así, al comprender cómo funciona el sistema de envase y embalaje y como éste se integra en el diseño del propio producto y de su cadena de suministro, es posible valorar el potencial de desarrollo del enfoque "Sustainable Packaging Logistics" (SPL).

Más aún, si se estructurase organizativamente este enfoque con un modelo que, sistemática y objetivamente, persiguiese la búsqueda de alternativas eficientes de envases y embalajes, se lograría materializar este objetivo de mejora en el comportamiento sostenible, tanto de empresas, como de cadenas de suministro.

Así, el objetivo empírico de este artículo (desarrollar y validar conceptual y empíricamente hasta qué punto la aplicación de un modelo estructurado que despliegue el enfoque SPL puede contribuir de forma activa a un mejor comportamiento sostenible en el conjunto de la cadena de suministro) ha sido desarrollado y parcialmente validado en el sector de menaje. 


\section{REFERENCIAS}

Accorsi, R., Cascini, A., Cholette, S., Manzini, R. y Mora, C. (2014), "Economic and environmental assessment of reusable plastic containers: a food catering case study", International Journal of Production Economics, Vol. 152, pp. 88-101.

AECOC, (2012), "Recomendaciones AECOC para la Logística. Unidades de carga eficientes", AECOC, Barcelona (Spain).

Albrecht, S., Brandstetter, P., Beck, T., Fullana-Palmer, P., Grönman, K., Baitz, M. y Fischer, M. (2013), “An extended life cycle analysis of packaging systems for fruit and vegetable transport in Europe", The International Journal of Life Cycle Assessment, Vol. 18 No 8, pp. 1549-1567.

Azzi, A., Battini, D., Persona, A. y Sgarbossa, F. (2012), "Packaging design: general framework and research agenda", Packaging Technology and Science, Vol. 25 No 8, pp. 435-456.

Bertoluci, G., Leroy, Y. y Olsson, A. (2014), “Exploring the environmental impacts of olive packaging solutions for the European food market", Journal of Cleaner Production, Vol. 64, pp. 234-243.

Christopher, M. (2005), Logistics and Supply Chain management strategies for reducing cost and improving service, Financial Times Pitman Publishing, London.

De Koeijer, B., Wever, R. y Henseler, J. (2017), "Realizing product-packaging combinations in circular systems: shaping the research agenda", Packag. Technol. Sci., 30 (8) (2017), pp. 443-460. (1)

De Koeijer, B., De Lange, J. y Wever, R. (2017), “Desired, Perceived, and Achieved Sustainability: Trade-Offs in Strategic and Operational Packaging Development", Sustainability, 9, 1923. (2)

European Commission (1994), Directive 94/62/EC on Packaging and Packaging Waste.

European Commission (2004), Directive 2004/12/EC on Packaging and Packaging Waste.

Freichel, S.L.K., Wollenburg, J. y Wörtge, J.K. (2020), "The role of packaging in omni-channel fashion retail supply chains: how can packaging contribute to logistics efficiency?", Logistics Research, Vol. 13 No. 1, pp. 1-20.
García-Arca, J. y Prado-Prado, J.C. (2008), "Packaging design model from a supply chain approach", Supply Chain Management: An International Journal, Vol. 13 No. 5, pp. 375-380.

García-Arca, J., Prado-Prado, J.C. y González-Portela Garrido, A.T. (2014), "Packaging logistics: promoting sustainable efficiency in supply chains", International Journal of Physical Distribution and Logistics Management, Vol. 44 No. 4, pp. 325-346.

García-Arca, J., González-Portela Garrido, A. T. y PradoPrado, J.C. (2016), "Packaging Logistics for improving performance in supply chains: the role of meta-standards implementation", Production, Vol. 26 No. 2, pp. 261272.

García-Arca, J., González-Portela Garrido, A. T. y PradoPrado, J.C. (2017), "Sustainable Packaging Logistics. The link between Sustainability and Competitiveness in Supply Chains", Sustainability, Vol. 9 No. 7, pp: 1098.

García-Arca, J., Trinidad González-Portela, A. y Carlos Prado-Prado, J. (2019), "Organizational best practices in packaging design. An analysis in perfumery and cleaning products", Direccion y Organizacion, Vol. 68, pp. 5-19.

García-Arca, J., Comesaña-Benavides, J.A., GonzálezPortela Garrido, A.T. y Prado-Prado, J.C. (2020), "Rethinking the Box for Sustainable Logistics". Sustainability, 12, 1870.

Gelici-Zeko, M. M., Lutters, D., Klooster, T. R., y Weijzen, P. L. G. (2012). Studying the influence of packaging design on consumer perceptions (of dairy products) using categorizing and perceptual mapping. Packaging Technology and Science, 26(4), 215-228.

Grönman, K., Soukka, R., JärviKääriäinen, T., Katajajuuri, J.-M., Kuisma, M., Koivupuro, H.-K., Ollila, M., Pitkänen, M., Miettinen, O., Silvenius, F., Thun, R., Wessman, H. y Linnanen, L. (2013), "Framework for Sustainable Food Packaging Design", Packaging Technology and Science, Vol. 26, pp. 187-200.

Hellström, D. y Saghir, M. (2007), "Packaging and logistics interactions in retail supply chains", Packaging Technology and Science, Vol. 20, pp. 197-216.

Hellström, D. y Nilsson, F. (2011), "Logistics-driven packaging innovation: A case study at IKEA", International Journal of Retail and Distribution Management, Vol. 39, pp. 638-657. 
Hellström, D. y Olsson, A. (2017), “Managing packaging design for sustainable development”, Wiley, London.

ISO (2006), "ISO 14044: 2006. Gestión ambiental. Análisis de ciclo de vida. Requisitos y directrices", Editado por ISO (Ginebra).

Klevas, J. (2005), "Organization of packaging resources at a product-developing company", International Journal of Physical Distribution and Logistics Management, Vol. 35 No. 2, pp. 116-131.

Kye, D., Lee, J. y Lee, K. (2013), “The perceived impact of packaging logistics on the efficiency of freight transportation (EOT)", International Journal of Physical Distribution and Logistics Management, Vol. 43 No. 8, pp. 707-720.

Likert, R. (1932). "A technique for the measurement of attitude", Archives of Psychology, 140, 5-55.

Lindh, H., Williams, H., Olsson, A. y Wikström, F. (2016), "Elucidating the Indirect Contributions of Packaging to Sustainable Development: A Terminology of Packaging Functions and Features", Packaging Technology and Science, Vol. 29, pp. 225-246.

Magnusson, A., Olander, M., y Olsson, A. (2012). Finding Methods for Innovative Packaging Development: The Card Approach. Editado por la Universidad de Lund (Suecia).

Mejía-Argueta, C., Soto-Cardona, O. C.,Albán, G., Mauricio, H., y Moreno-Moreno, J. P. (2015), "Minimum cost package size analysis in the supply chain: A case study in Colombia", Estudios Gerenciales, 31(134), 111-121.

Molina-Besch, K. y Pålsson, H. (2014), "Packaging for Eco-Efficient Supply Chains: Why Logistics Should Get Involved in the Packaging Development Process", Sustainable Logistics (Transport and Sustainability), Vol. 6, pp. 137-163.

Molina-Besch, K. y Pålsson, H. (2020), “A simplified environmental evaluation tool for food packaging to support decision-making in packaging development", Packag Technol Sci, 33(4-5), 141-157

Morabito, R., Morales, S. R. y Widmer, J. A. (2000). Loading optimization of palletized products on trucks. Transportation Research Part E: Logistics and Transportation Review, 36(4), 285-296.
Näslund, D., Kale, R., Paulraj, A., (2010). “Action Research in supply chain management - a framework for relevant and rigorous research". Journal of Business Logistics. 31, 331-355.

Olander-Roese, M., y Nilsson, F. (2009). Competitive Advantage Through Packaging Design? Propositions for Supply Chain Effectiveness and Efficiency. In DS 58-1: Pro-ceedings of ICED 09, the 17th International Conference on Engineering Design, Vol. 1, Design Processes, Palo Alto, CA, USA, 24.-27.08. 2009.

Olsmats, C. y Dominic, C. (2003), "Packaging scorecard. A packaging performance evaluation method", Packaging Technology and Science, Vol. 16 No. 1, pp. 9-14.

Pålsson, H., Finnsgård, C. y Wänström, C. (2013), "Selection of packaging systems in supply chains from a sustainability perspective: the case of Volvo", Packaging Technology and Science, Vol. 26 No. 5, pp. 289-310.

Pålsson, H. y Hellström, D. (2016), "Packaging logistics in supply chain practice - current state, trade-offs and improvement potential", International Journal of Logistics Research and Applications, Vol. 19 No. 5, pp. 351-368.

Pålsson, H. (2018), "Packaging Logistics. Understanding and managing the economic and environmental impacts of packaging in supply chains", Kogan Page, London.

Pålsson, H. y Sandberg, E. (2020), "Paradoxes in supply chains: a conceptual framework for packed products", The International Journal of Logistics Management, Vol. 31 No. 3, pp. 423-442

Rundh, B. (2016), "The role of packaging within marketing and value creation", British Food Journal, Vol. 118 No. 10, pp. 2491-2511.

Saghir, M. (2002), Packaging Logistics Evaluation in the Swedish Retail Supply Chain, Lund University, Lund.

Seuring, S. y Müller, M. (2008), "From a literature review to a conceptual framework for sustainable supply chain management", Journal of Cleaner Production, Vol. 16, 1699-1710.

Siracusa, V., Ingrao, C., Giudice, A. L., Mbohwa, C., y Dalla Rosa, M. (2014). Envi-ronmental assessment of a multilayer polymer bag for food packaging and preservation: An LCA approach. Food Research International, $62,151-161$ 
Sohrabpour, V., Hellström, D. y Jahre, M. (2012), "Packaging in developing countries: identifying supply chain needs", Journal of Humanitarian Logistics and Supply Chain Management, Vol. 2 No. 2, pp. 183- 205.

Sohrabpour, V., Oghazi, P. y Olsson, A. (2016), “An Improved Supplier Driven Packaging Design and Development Method for Supply Chain Efficiency", Packaging Technology Science, Vol. 29, pp. 161-173.

Svanes, E., Vold, M., Møller, H., Pettersen, M.K., Larsen, H. y Hanssen, O.J. (2010), "Sustainable packaging design: a holistic methodology for packaging design", Packaging Technology and Science, Vol. 23 No. 3, pp. 161-175.

Verghese, K., y Lewis, H. (2007). "Environmental innovation in industrial packaging: a supply chain approach", International Journal of Production Research, 45(1819), 4381-4401.

Vernuccio, M., Cozzolino, A. y Michelin, I.L. (2010), “An exploratory study of marketing, logistics, and ethics in packaging innovation", European Journal of Innovation Management, Vol. 13, pp. 333-354.
Wever, R. (2011). Design for volume optimization of packaging for durable goods. Packaging Technology and Science, 24(4), 211-222.

Wever, R., y Vogtländer, J. (2013). Eco-efficient Value Creation: An Alternative Per-spective on Packaging and Sustainability. Packaging Technology and Science, 26(4), 229-248.

Wikström, F.; Verghese, K., Auras, R., Olsson, A., Williams, H., Wever, R., Grönman, K., Kvalvåg Pettersen, M., Møller, H. y Soukka, R. (2018), "Packaging Strategies That Save Food: A Research Agenda for 2030”. J. Ind. Ecol.,14, 1346.

Williams, H., Wikström, F. y Löfgren, M. (2008). A life cycle perspective on environ-mental effects of customer focused packaging development. Journal of Cleaner Pro-duction vol. 16, pp. 853-859.

Williams, H., y Wikström, F. (2011). Environmental impact of packaging and food loss-es in a life cycle perspective: a comparative analysis of five food items. Journal of Cleaner Production, 19(1), 43-48. 